\section{ESTILOS DE APRENDIZAJE Y ESTILOS DE PENSAMIENTO EN ESTUDIANTES UNIVERSITARIOS. APRENDER A APRENDER Y APRENDER A PENSAR}

\author{
Pedro Allueva Torres \\ Departamento de Psicología y Sociología, \\ ICE-Universidad de Zaragoza \\ C/ Pedro Cerbuna, 12, 50009 Zaragoza, España \\ pallueva@unizar.es \\ Concepción Bueno García \\ Departamento de Ciencias de la Educación, \\ ICE-Universidad de Zaragoza \\ C/ Pedro Cerbuna, 12, 50009 Zaragoza, España \\ cbueno@unizar.es
}

\begin{abstract}
The questionnaire Honey-Alonso about Learning Styles (CHAEA) and the questionnaire Sternberg-Wagner about Thinking Styles were passed to a sample of first-year students of the different Degree of the University of Zaragoza with the aim of being able to determine their kind of learning styles and thinking styles. Moreover, data were analysed in order to study the potential correlation between the Learning Styles and the Thinking Styles of the aforementioned questionnaires. The knowledge of the learning and thinking styles will facilitate students their metacognitive knowledge, as well as helping them to learn to learn and to learn to think, facilitating in such way the learning process of the different subjects and the development of learning and thinking abilities.
\end{abstract}

KEY WORDS: Learning styles; thinking styles; learning to learn; learning to think; development of learning abilities; development of thinking abilities.

\section{INTRODUCCIÓN}

Como educadores la mejor enseñanza que podemos -debemos realizar con los estudiantes es la de enseñarles a aprender y enseñarles a pensar. $Y$ como estudiantes el mejor aprendizaje que pueden hacer es el de aprender a aprender y aprender a pensar. No es tan importante la cantidad de información que es capaz de recordar una persona como la habilidad de saber qué información le falta, cómo adquirirla y qué estrategias utilizar en el tratamiento de dicha información. Asimismo, conocer qué

\section{LEARNING STYLES AND THINKING STYLES OF UNIVERSITY STUDENTS. LEARNING TO LEARN AND LEARNING TO THINK}

RESUMEN: Con el fin de poder determinar con qué estilos de aprendizaje y estilos de pensamiento llegan los estudiantes a las distintas titulaciones a la Universidad de Zaragoza, se les pasó, a una muestra de estudiantes de $1 .^{\circ}$ curso, el Cuestionario Honey-Alonso de Estilos de Aprendizaje (CHAEA) y el Cuestionario de Estilos de Pensamiento de Sternberg-Wagner. Asimismo, se realizaron análisis de los datos para estudiar las posibles correlaciones existentes entre los Estilos de Aprendizaje y los Estilos de Pensamiento de dichos cuestionarios. El conocimiento de los estilos de aprendizaje y pensamiento por parte de los estudiantes les facilitará el conocimiento metacognitivo, así como, que aprendan a aprender y aprendan a pensar, facilitando asi el proceso de aprendizaje de las distintas materias y el desarrollo de habilidades de aprendizaje y del pensamiento.

PALABRAS CLAVE: Estilos de aprendizaje; estilos de pensamiento; aprender a aprender; aprender a pensar; desarrollo de habilidades de aprendizaje y desarrollo de habilidades del pensamiento.

habilidades del pensamiento posee, cómo desarrollarlas y cómo utilizarlas en los distintos momentos y situacionesproblema de la vida es más importante que sobresalir en alguna de las aptitudes del pensamiento. Para Allueva (2011), "Saber pensar implica saber utilizar de forma adecuada los conocimientos, las aptitudes y habilidades del pensamiento, así como, los recursos cognitivos que tiene la persona. Saber pensar implica utilizar adecuadamente tanto el pensamiento convergente como el pensamiento divergente, desde el control y regulación del conocimiento metacognitivo." ... "Aprender a pensar y enseñar a pensar, 
debe ser un objetivo prioritario del profesorado y demás profesionales relacionados con el ámbito educativo" ( $p$. 4563). Para aprender a aprender y para aprender a pensar hay un denominador común que es el conocimiento metacognitivo. El conocimiento de su Perfil de Estilos de Aprendizaje y de Estilos de Pensamiento aumentará dicho conocimiento metacognitivo. A partir de esta afirmación nos planteamos algunos interrogantes: ¿Qué relación existe entre el Perfil de Estilos de Aprendizaje y el Perfil de Estilos de Pensamiento? ¿Los Cuestionarios de Estilos de Aprendizaje y de Pensamiento, miden estilos de aprendizaje/pensamiento similares o miden estilos de aprendizaje/pensamiento distintos? De forma resumida la pregunta que nos planteamos es si ¿existe correlación lineal entre los Estilos de Aprendizaje y los Estilos de Pensamiento?

Para llevar a cabo el presente estudio hemos estimado conveniente partir de la Teoría de Estilos de Aprendizaje de Honey-Alonso y del Cuestionario Honey-Alonso de Estilos de Aprendizaje (CHAEA), por estar ampliamente validado. Asimismo, en lo referente a Estilos de Pensamiento, hemos considerado oportuno partir de la Teoría del Autogobierno Mental de Sternberg y del Cuestionario de Estilos de Pensamiento de Sternberg-Wagner basado en dicha teoría y de validez contrastada.

Así, el problema que nos planteamos en esta investigación es el siguiente: "¿existe correlación lineal entre el Perfil de Estilos de Aprendizaje de Honey-Alonso y el Perfil de Estilos de Pensamiento de Sternberg?".

Además de conocer las posibles correlaciones existentes entre dichos Perfiles de Estilos de Aprendizaje y de Pensamiento, otro objetivo que nos planteamos con la presente investigación es "Conocer el Perfil de Estilos de Aprendizaje y de Estilos de Pensamiento por titulaciones que predominan en los estudiantes de primer curso de la Universidad de Zaragoza".

Para definir los Estilos de Aprendizaje, tomaremos las palabras de Alonso, Gallego y Honey (2005), "Desde nuestro punto de vista, una de las definiciones más claras y ajustadas es la que propone Keefe (p. 48) y que hacemos nuestra: Los estilos de aprendizaje son los rasgos cognitivos, afectivos y fisiológicos que sirven como indicadores relativamente estables, de cómo los alumnos perciben interacciones y responden a sus ambientes de aprendizaje".
A partir de CHAEA: Cuestionario Honey-Alonso de Estilos de Aprendizaje, se obtiene un Perfil de cuatro Estilos de Aprendizaje. Siguiendo a Alonso, Gallego y Honey (2005), nombramos las características principales de los cuatro Estilos de Aprendizaje: A) Las personas que obtengan un predominio claro del Estilo Activo poseerán algunas de estas características o manifestaciones: Animador, improvisador, descubridor, arriesgado, espontáneo. B) Las personas que obtengan un predominio claro del Estilo Reflexivo poseerán muchas de estas características o manifestaciones: Ponderado, concienzudo, receptivo, analítico, exhaustivo. C) Las personas que obtengan una mayor puntuación en el Estilo Teórico tendrán características o manifestaciones como éstas: Metódico, lógico, objetivo, crítico, estructurado. D) Las personas que obtengan una mayor puntuación en el Estilo Pragmático tendrán características o manifestaciones como éstas: Experimentador, práctico, directo, eficaz, realista.

Para definir el Estilo de Pensamiento tomamos la definición que nos da Sternberg (1999): "Un estilo es una manera característica de pensar. No se refiere a una aptitud, sino a cómo utilizamos las aptitudes que tenemos. No tenemos un estilo, sino un perfil de estilos. Las personas pueden ser prácticamente idénticas en cuanto a sus aptitudes y aun así tener estilos muy distintos" (p. 38). El Cuestionario de Estilos de Pensamiento de Sternberg-Wagner está basado en la Teoría del Autogobierno Mental de Sternberg, 1999, comprendiendo los estilos de pensamiento Legislativo, Ejecutivo y Judicial: A) "A las personas legis/ativas les gusta hacer las cosas a su aire y prefieren decidir por sí mismas qué harán y cómo lo harán. A las personas legislativas les gusta establecer sus propias reglas y prefieren los problemas que no estén estructurados o planteados de antemano. B) A las personas ejecutivas les gusta seguir reglas y prefieren los problemas estructurados y planteados de antemano. Les gusta rellenar las lagunas de estructuras ya existentes en vez de crear estructuras ellas mismas. C) A las personas judiciales les gusta evaluar reglas y procedimientos, y prefieren problemas donde se analicen y evalúen cosas e ideas ya existentes" (p. 49).

\section{Metodología}

En el primer trimestre del curso 2008-2009, 811 estudiantes de primer curso de la Universidad de Zaragoza 
cumplimentaron el Cuestionario de Estilos de Pensamiento de Sternberg-Wagner, en Allueva, Herrero y Franco (2010). Asimismo, en el primer trimestre de los cursos 2008-2009, 2009-2010 y 2010-2011, 464 estudiantes de primer curso de la Universidad de Zaragoza cumplimentaron el Cuestionario Honey-Alonso de Estilos de Aprendizaje (CHAEA).
En ambos casos, los estudiantes estaban matriculados en las mismas 12 titulaciones, impartidas en siete centros de tres campus de la Universidad de Zaragoza. En la Tabla 1, puede observarse la distribución de participantes por frecuencia y porcentaje en las 12 titulaciones según Estilos de Aprendizaje y Estilos de Pensamiento.

TABLA 1: DISTRIBUCIÓN DE FRECUENCIAS POR TITULACIONES SEGÚN ESTILOS DE APRENDIZAJE Y DE PENSAMIENTO

\begin{tabular}{|l|c|c|c|c|}
\hline \multirow{2}{*}{ Titulación } & \multicolumn{2}{|c|}{ E. Apr. (EA) } & Frec. & Porc. \\
\cline { 2 - 5 } & Frec. & Porc. & 162 & 20,0 \\
\hline Adm. y Dir. Empr. & 115 & 24,8 & 145 & 17,9 \\
\hline Económicas & 34 & 7,3 & 12 & 1,5 \\
\hline Geología & 3 &, 6 & 20 & 2,5 \\
\hline Matemáticas & 13 & 2,8 & 31 & 3,8 \\
\hline Química & 11 & 2,4 & 65 & 8,0 \\
\hline Magist. E. Esp. & 12 & 2,6 & 65 & 8,0 \\
\hline Magist. E. Física & 6 & 1,3 & 98 & 12,1 \\
\hline Magist. Infantil & 24 & 5,2 & 40 & 4,9 \\
\hline Magist. L. Extran. & 16 & 3,4 & 59 & 7,3 \\
\hline Magist. Primaria & 50 & 10,8 & 7 & 9 \\
\hline Relaciones Labo. & 49 & 10,5 & 107 & 13,2 \\
\hline Trabajo Social & 131 & 28,2 & $\mathbf{8 1 1}$ & 100,0 \\
\hline Total & 464 & 100,0 & & 9 \\
\hline
\end{tabular}

Para calcular las medias de los valores obtenidos en los Cuestionarios de Estilos de Aprendizaje y de Estilos de Pensamiento, se han agrupado los participantes en cuatro bloques en función de las titulaciones. El primer bloque está formado por las titulaciones de Económicas y Administración y Dirección de Empresas (ADE), el segundo bloque, que hemos denominado Ciencias, está formado por las titulaciones de Geología, Matemáticas y Químicas, el tercer bloque agrupa las cinco titulaciones de Magisterio y el cuarto bloque está formado por las titulaciones de Relaciones Laborales y Trabajo Social. Ver tabla 2.

Para dar respuesta al problema planteado en la investigación se han calculado las Correlaciones y Significación Bilateral entre los valores totales obtenidos en los Cuestionarios de Estilos de Aprendizaje y de Estilos de Pensamiento.

\section{Resultados}

En la tabla 2 y en los gráficos 1 y 2, se presentan las medias de los valores obtenidos en los Cuestionarios de Estilos de Aprendizaje y de Estilos de Pensamiento por bloques de titulaciones. En los cuatro bloques de titulaciones, obtiene una mayor puntuación el Estilo de Aprendizaje Reflexivo, seguido de los Estilos Teórico, Pragmático y Activo respectivamente. En las puntuaciones obtenidas en el Cuestionario de Estilos de Pensamiento, observamos cómo en los bloques de las titulaciones de Ciencias, Magisterio y, Relaciones Laborales y Trabajo Social obtiene mayor puntuación el Estilo Legislativo seguido de los Estilos Ejecutivo y Judicial respectivamente. En el bloque de titulaciones de Económicas y ADE es el Estilo Ejecutivo el que obtiene la puntuación media más alta seguido de los Estilos Legislativo y Judicial respectivamente.

ARBOR Vol. 187 Extra 3 diciembre [2011] 261-266 ISSN: 0210-1963 
TABLA 2: MEDIAS DE LOS ESTILOS DE APRENDIZAJE Y DE PENSAMIENTO EN CADA UNO DE LOS BLOQUES DE TITULACIONES

\begin{tabular}{|c|c|c|c|c|c|c|c|c|}
\hline \multirow{2}{*}{$\begin{array}{l}\text { Titulaciones } \\
\text { por Bloques }\end{array}$} & & \multicolumn{3}{|c|}{ Estilos de Aprendizaje (EA) } & \multirow[b]{2}{*}{ Pragmático } & \multicolumn{3}{|c|}{ Estilos de Pensamiento (EP) } \\
\hline & & Activo & Reflexivo & Teórico & & Legislativo & Ejecutivo & Judicial \\
\hline \multirow[t]{3}{*}{ Económ./ADE } & Media & 10,70 & 15,01 & 13,36 & 12,59 & 40,31 & 40,70 & 36,71 \\
\hline & N & 149 & 149 & 149 & 149 & 307 & 307 & 307 \\
\hline & Desv. típ. & 3,715 & 2,994 & 2,892 & 2,771 & 5,762 & 5,870 & 5,919 \\
\hline \multirow[t]{3}{*}{ Ciencias } & Media & 11,26 & 14,81 & 13,30 & 12,22 & 40,65 & 38,37 & 37,02 \\
\hline & $\mathrm{N}$ & 27 & 27 & 27 & 27 & 63 & 63 & 63 \\
\hline & Desv. típ. & 3,347 & 2,732 & 2,906 & 2,259 & 7,021 & 5,703 & 5,422 \\
\hline \multirow[t]{3}{*}{ Magisterio } & Media & 11,68 & 14,54 & 12,81 & 12,46 & 41,10 & 38,83 & 35,84 \\
\hline & N & 108 & 108 & 108 & 108 & 327 & 327 & 327 \\
\hline & Desv. típ. & 3,177 & 3,446 & 2,999 & 2,470 & 6,128 & 6,538 & 6,138 \\
\hline \multirow{3}{*}{$\begin{array}{l}\text { R. Laborales/ } \\
\text { T. Social }\end{array}$} & Media & 12,13 & 14,66 & 12,39 & 12,26 & 40,86 & 39,64 & 37,91 \\
\hline & $\mathrm{N}$ & 180 & 180 & 180 & 180 & 114 & 114 & 114 \\
\hline & Desv. típ. & 3,333 & 2,977 & 3,039 & 2,825 & 6,581 & 5,749 & 5,927 \\
\hline \multirow[t]{3}{*}{ Total } & Media & 11,52 & 14,75 & 12,85 & 12,41 & 40,73 & 39,62 & 36,55 \\
\hline & $\mathrm{N}$ & 464 & 464 & 464 & 464 & 811 & 811 & 811 \\
\hline & Desv. típ. & 3,470 & 3,081 & 2,996 & 2,694 & 6,131 & 6,175 & 6,003 \\
\hline
\end{tabular}

Las Correlaciones y Significación Bilateral calculadas a partir de los valores totales obtenidos en los Cuestionarios de Estilos de Aprendizaje y de Estilos de Pensamiento (ver tabla 3), nos muestran que no hay correlación lineal significativa entre ninguno de los Estilos de Aprendizaje y Estilos de Pensamiento.

\section{Discusión}

\section{A. Interpretación de los resultados}

Al analizar las medias de los datos obtenidos en los Perfiles de Estilos de Aprendizaje por bloques de titulaciones, observamos que hay pequeñas diferencias en el perfil de estilos que tienen los estudiantes de primer curso que acceden a la Universidad de Zaragoza. De forma similar sucede con los Perfiles de Estilos de Pensamiento, con la salvedad del Bloque de las titulaciones de Económicas y ADE que puntúan más alto en el Estilo Ejecutivo seguido del Legislativo y Judicial. Lógicamente, en la individualidad y personalización de los estudiantes en el aula nos encon- tramos con diferentes perfiles de estilos de Aprendizaje y de Pensamiento que deberemos tener en cuenta tanto en la metodología como en el sistema de evaluación utilizados. La falta de correlación lineal entre los Estilos de Aprendizaje y de Pensamiento encontrados, quiere decir que ambos cuestionarios dan una información distinta y complementaria al estudiante.

\section{B. Implicaciones Educativas}

Para un mayor y mejor conocimiento metacognitivo es conveniente que los estudiantes conozcan cuáles son sus Perfiles de Estilos de Aprendizaje y de Pensamiento. Este conocimiento contribuirá muy positivamente en el desarrollo de estrategias cognitivas y metacognitivas, y para aprender a aprender y aprender a pensar, y les será especialmente útil para saber cómo afrontar las distintas tareas de aprendizaje. Dado que los cuatro Estilos de Aprendizaje obtenidos a partir del Cuestionario de Honey-Alonso (CHAEA) y los tres Estilos de Pensamiento obtenidos a partir del Cuestionario de Sternberg-Wagner se complementan, dando una información más completa de la forma de aprender y pensar de los estudiantes, será conveniente 


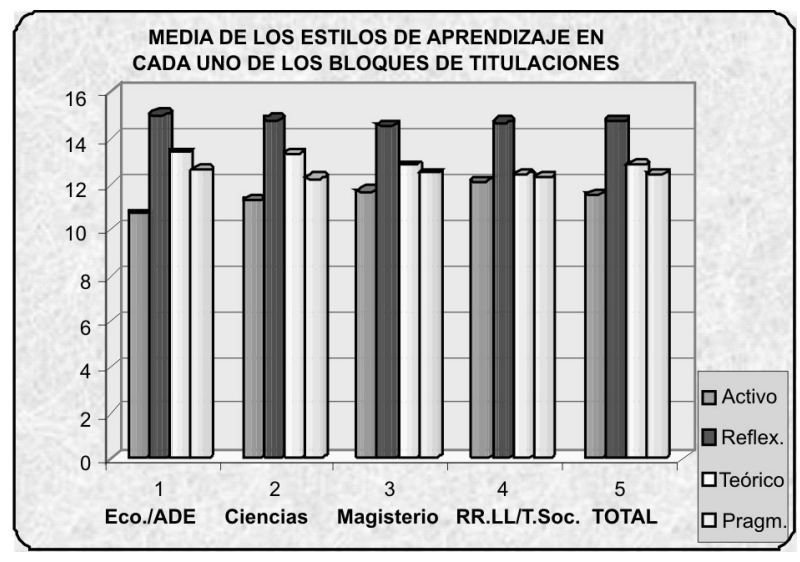

Gráfico 1. Media de los Estilos de Aprendizaje en cada uno de los bloques de titulaciones.

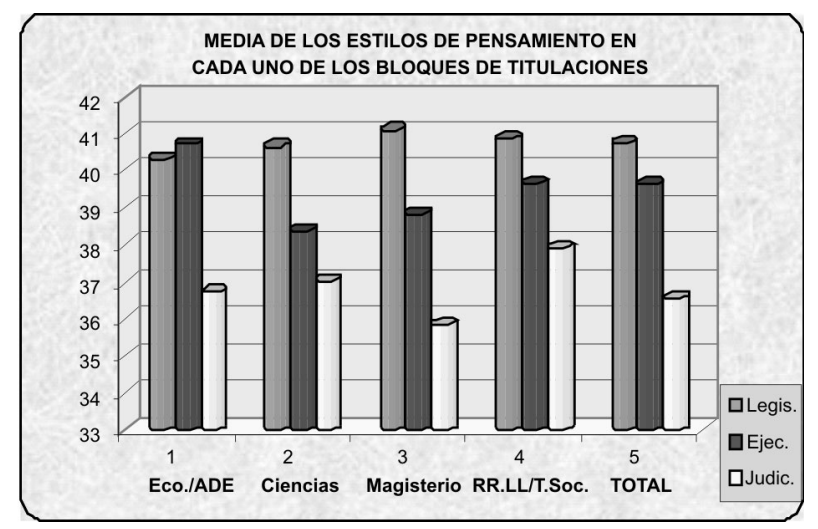

Gráfico 2. Media de los Estilos de Pensamiento en cada uno de los bloques de titulaciones.

TABLA 3: CORRELACIONES Y SIGNIFICACIÓN BILATERAL ENTRE LOS ESTILOS DE APRENDIZAJE Y DE PENSAMIENTO

\begin{tabular}{|c|c|c|c|c|c|c|}
\hline & & & \multicolumn{4}{|c|}{ Estilos de Aprendizaje (EA) } \\
\hline & & & Activo & Reflexivo & Teórico & Pragmático \\
\hline \multirow{6}{*}{$\begin{array}{c}\text { Estilos de } \\
\text { Pensamiento (EP) }\end{array}$} & \multirow{2}{*}{ Ejecutivo } & Corr. Pearson &, 328 & ,448 &,- 206 &,- 447 \\
\hline & & Sig. (bilateral) &, 298 &, 144 &, 521 & .145 \\
\hline & \multirow[t]{2}{*}{ Legislativo } & Corr. Pearson & .252 &, 045 &,- 280 &, 166 \\
\hline & & Sig. (bilateral) & .429 & 891 &, 378 & 606 \\
\hline & \multirow[t]{2}{*}{ Judicial } & Corr. Pearson &, 257 & .453 &,- 483 &,- 395 \\
\hline & & Sig. (bilateral) & 421 & 139 & ,112 & ,204 \\
\hline
\end{tabular}

que realicen dichos cuestionarios recibiendo la información y formación necesarias, por parte de especialistas, para que les pueda ser útil en su proceso de aprendizaje y desarrollo. De acuerdo con Kaufman \& Grigorenko, 2009, hay personas a las que les gusta pensar de una determinada manera, pero no piensan así, asimismo puede suceder lo contrario. Dependiendo de los estilos de aprendizaje y pensamiento predominantes en los estudiantes, éstos lograrán un mayor rendimiento cuando las metodologías y los sistemas de evaluación utilizados en las asignaturas se asemejen más a dichos perfiles de estilos predominantes. Por otra parte, cada profesor/a tiene un perfil de estilos de pensamiento en el que se encuentra más cómodo a la hora de explicar e incluso a la hora de planificar metodológi- camente las materias que tenga que impartir en el aula, pero de acuerdo con Michavila (2009), "Debe conseguirse que, en definitiva, la prioridad la tenga el aprendizaje, más allá de la enseñanza. Hay que interesarse más por aquello que aprende el estudiante que por aquello que enseña el profesor" (p. 5). Por tanto, el profesorado más allá de sus preferencias, según su perfil de estilos de pensamiento predominante, debe conseguir llegar a todos los estudiantes desde los diferentes estilos, metodologías y sistemas de evaluación, de tal forma que los estudiantes sean capaces de desarrollar estrategias de trabajo para los diferentes estilos de aprendizaje y pensamiento logrando un mayor y mejor aprendizaje. Asimismo, según la materia, el profesorado debe utilizar y enseñar a trabajar con las

ARBOR Vol. 187 Extra 3 diciembre [2011] 261-266 ISSN: 0210-1963 
metodologías más acordes con los estilos de aprendizaje y de pensamiento más adecuados para esa materia. Para Allueva, Herrero y Franco (2010), "Tanto el estilo de pensamiento del alumnado como el del profesorado deben ser flexibles, de forma que sean capaces de adaptarse a distintos tipos de procesos de enseñanza-aprendizaje" (p. 238). Es decir, hay que llegar a todos los estudiantes desde los distintos estilos de aprendizaje y pensamiento, pero también debe incidirse especialmente en los estilos de aprendizaje y pensamiento que favorezcan el aprendizaje de las diferentes materias. De tal forma que los estudiantes desarrollen distintas estrategias y habilidades cognitivas y metacognitivas, que les ayuden a aprender a aprender y a aprender a pensar, y así poder transferirlas a distintas situaciones de aprendizaje y desarrollo. Como decía Vygotski (1996), "El buen aprendizaje es sólo aquel que precede al desarrollo" (p. 138).

\section{Conclusiones}

Los Estilos de Aprendizaje y de Pensamiento, obtenidos a partir de los Cuestionarios de Honey-Alonso (CHAEA) y de Sternberg-Wagner respectivamente, se complementan dando una información más completa.

El conocimiento obtenido por parte de los estudiantes, a partir de dichos cuestionarios, de su forma de aprender y pensar les ayudará a desarrollar habilidades de aprendizaje y de pensamiento favoreciendo el proceso de aprendizaje. Asimismo, que el profesorado conozca el Perfil de Estilos de Aprendizaje y de Pensamiento de los estudiantes facilitará que realice un diseño metodológico y de sistema de evaluación que estimule y favorezca dicho proceso de aprendizaje y desarrollo en los estudiantes, de forma que aprendan a aprender y aprendan a pensar.

\section{BIBLIOGRAFÍA}

Allueva, P.; Herrero, M. L. y Franco, J. A. (2010): "Estilo de pensamiento del alumnado y profesorado universitario. Implicaciones educativas", REIFOP, 13 (4), pp. 227-240.

Allueva, P. (2011): "Aprender a pensar y enseñar a pensar. Proceso de resolución de problemas", en J. M. Román, M. A. Carbonero y J. D. Valdivieso (comp.), Educación, aprendizaje y desarrollo en una sociedad multicultural, Madrid, Asociación de Psicología y Educación, pp. 4563-4572.

Alonso, C. M.; Gallego, D. J. y Honey, P. (2005): Los estilos de aprendizaje. Procedimientos de diagnóstico y mejora, Ediciones Mensajero, Bilbao.

Kaufman, J. C. y Grigorenko, E. L. (eds.) (2009): The essential Sternberg: essays on intelligence, psychology, and edu- cation, New York, Springer Publishing Company, LLC.

Keefe, J. W. (1998): "Profiling and Utilizing Learning Style", NASSP, Reston, Virginia, en C. M. Alonso, D. J. Gallego y P. Honey (2005): Los estilos de aprendizaje. Procedimientos de diagnóstico y mejora, Ediciones Mensajero, Bilbao.

Michavila, F. (2009): "La Innovación Educativa. Oportunidades y Barreras", ARBOR Ciencia, Pensamiento y Cultura. Consejo Superior de Investigaciones Científica, vol. 185, n. ${ }^{\circ}$ Extra, pp. 3-8.

Sternberg, R. J. (1999): Estilos de pensamiento. Claves para identificar nuestro modo de pensar y enriquecer nuestra capacidad de reflexión, Paidós, Barcelona.

Vygotski, L. S. (1996): El desarrollo de los procesos psicológicos superiores, Crítica, Barcelona. 